\title{
Optimum Interpolation of Imaging Microwave Radiometer Data
}

\author{
GENE A. POE
}

\begin{abstract}
A technique to interpolate imaging microwave radiometer data is presented as an application of the Backus-Cilhert theory. The interpolation process is optimal in the sense that it attempts to preserve the spatial resolution of the antenna gain function associated with the sampled radiometer data. The technique is applied to the Special Sensor Microwave/Imager (SSM/I) satellite data, and is found to enhance the high-resolution features of the imagery. The technique is expected to be a useful tool in creating images for detailed analysis.
\end{abstract}

\section{INTRODUCTION}

$I^{\mathrm{N}}$ $\mathrm{N}$ many situations, proper imaging of microwave radiometer data requires an antenna scan rate and associated data sampling ratc consistent with collecting all the spatial frequency information sensed by the antenna. If the antenna is treated as having a band-limited spatial frequency response $W \mathrm{~km}^{-1}[1]$, then the scan and sampling rates should be selected to be greater than or cqual to the minimum sampling rate $2 W$ samples per $\mathrm{km}$ often referred to as the Nyquist ratc [2]. Duc to sampling limitations, the amount of data collected may be inadequate to conduct detailed image analysis. In addition, the spatially sampled grid may not be compatible with the desired image projection coordinates. Thus, interpolating, resampling, or smoothing of the radiometric data may bccomc important parts of the image processing function.

It is, of course, desirable that the interpolation process preserve the spatial resolution of the sampled radiometric data as well as avoid introducing data processing artifacts that could alter or destroy the high spatial frequency content of the image. In addition, the interpolation should avoid degrading the radiometric image with excessive amplification of instrument noise appearing in the sampled data.

The interpolation filter, as it is commonly referred to, may be viewed as "optimal" if the rcsulting interpolated data correspond to what would have been measured had the radiometcr actually made the measurements. Certainly the resulting interpolated data would possess the spatial resolution of the orginally sampled data, avoid amplification of sensor noise. and, in addition, retain any peculiar features associated with the antenna spatial frequency response (e.g., beam assymetry) or with the data

Manuscript receıved July 17, 1989; revised September 21, 1989

The author was with the Space Sensing Branch. Naval Center for Space Technology, Naval Research Laboratory, Washington, DC 20375-5000. $\mathrm{He}$ is now with Acrojet ElectroSystems, 1100 West Hollyvale Street. P.O Box 296. Azusa, CA 91702

IEEE Log Number 9037460 sampling geometry. The intent of this paper is to show that the Backus-Gilbert theory may be applied to closely approximate an optimal linear interpolation filter for imaging microwave radiometer data.

The paper draws upon the theoretical results presented by Stogryn [3] who applied the Backus-Gilbert methodology to attempt beam shaping of the antenna gain function at the sampled data points as well as remove crosspolarization coupling. Although the emphasis here is on interpolation, the mathematics of the Backus-Gilbert appreach is quite general and may be applied to a number of image processing problems. For example, Stogryn [3] notes the application to smoothing a high spatial resolution image to match the resulting resolution to that of a specified lower resolution image, and [4] shows the improvement in retrieving sea surface temperature from multifrequency radiometer data when commensurate spatial resolutions are maintained in the retrievals.

This paper does not address the subject of increasing the spatial resolution of the interpolated radiometer data beyond that associated with the antenna $3 \mathrm{~dB}$ beam diarneter. Attempts to extract frequency information from an image beyond the standard diffraction limits of the antenna aperture and obtain higher resolution (i.e., "superresolution") image features has received considerable attention in the literature [5]-[7]. Unfortunately, most methods that offer higher spatial resolution incur relatively large amplification of sensor noise and disturbing artifacts in the processed image.

\section{THEORY}

Consider a scanning microwave radiometer sensing the earth's upwelling brightness temperature field shown in Fig. 1. The sampled radiometer output at time $t$ expressed as an antenna temperature $T_{A}$ provides a measurement of the brightness temperature $T_{B}$ incident on the antenna, and it may be expressed as

$$
\begin{aligned}
T_{A}\left(\hat{s}_{A}(t), t\right)= & \int_{-\infty}^{\infty} d t^{\prime} h\left(t-t^{\prime}\right) \\
& \cdot \int_{E} d \Omega G\left(\hat{s}_{o}\left(t^{\prime}\right), \hat{s}\right) T_{B}\left(\vec{\rho}, \hat{s}, t^{\prime}\right)
\end{aligned}
$$

where $\hat{s}_{A}(t)$ is the effective pointing direction of the antenna associated with the radiometer sample at time $t, h$ is the impulse response of the radiometer receiver presampling low-pass filter, and $G$ is the antenna gain function

U.S. Government work not protected by U.S. copyright 


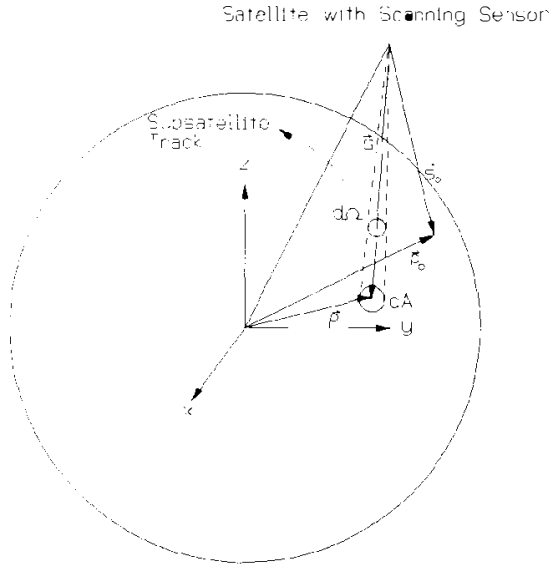

Fig. 1. Geometry and integration elements for antenna temperature (equation (1))

which describes the angular weighting of radiation incident on the antenna in direction of unit vector $-\hat{s}$ with the antenna in direction of unit vector $-\hat{s}$. It is assumed that $h$ is normalized so that $\int_{-\infty}^{\infty} d t h=1$, and that $G$ is sufficiently directive so that the angular integration may bc restricted to the earth's surface and $\int_{E} d \Omega G=1$. It is also assumed that $G$ has negligible cross-polararization coupling or that a correction has been made to remove its effect on $T_{A}$ (see [3]) so that (1) applies to the principal polarization of interest. In general, the earth's brightness tempcrature field $T_{B}$ depends on the direction $\hat{s}$, the position vector $\vec{\rho}$, as well as time. The effective pointing direction $\hat{s}_{A}(t)$ may be defined as

$$
\hat{s}_{A}(t)=\int_{-\infty}^{\infty} d t^{\prime} h\left(t-t^{\prime}\right) \hat{s}_{o}\left(t^{\prime}\right) .
$$

To simplify (1), the angular integration is transformed to an area integration on the surface of the earth, and the time and spatial integrations are interchanged to yield

$$
\begin{gathered}
T_{A}\left(\hat{s}_{A}(t), t\right)=\int_{E} d A \int_{-\infty}^{\infty} d t^{\prime}\left\{h ( t - t ^ { \prime } ) G \left(\hat{s}_{o}\left(t^{\prime}\right),\right.\right. \\
\left.\left.\hat{s}\left(t^{\prime}\right)\right)\left[\frac{-\hat{s}\left(t^{\prime}\right) \cdot \hat{\rho}}{s^{2}\left(t^{\prime}\right)}\right]\right\} \\
T_{B}\left(\vec{\rho}, \hat{s}\left(t^{\prime}\right), t^{\prime}\right)
\end{gathered}
$$

where unit vector $\hat{\rho}$ points toward area element $d A$ shown in Fig. 1. Expression (3) assumes an idealized spherical model for the earth. The quantity within the brackets in (3) may be identified as the effective areal weighting given by the antenna to the brightness temperature field associated with area $d A$ at position $\vec{\rho}$ in direction $\hat{s}\left(t^{\prime}\right)$ at time $t^{\prime}$.

To proceed much furthcr, additional information is needed concerning the directional and time dependence of $T_{B}$. Following the development in [3], it will be assumed that over the time period of interest, i.e., the measurement interval associated with the collection of radiometer data used in the interpolation processing, the brightness temperature upwelling from area $d A$ at $\vec{\rho}$ is essentially independent of direction $-\hat{s}$ over the angular regions of interest, and the time variation of $T_{B}$ may be neglected:

$$
T_{B}\left(\vec{\rho}, \hat{s}\left(t^{\prime}\right), t^{\prime}\right)=T_{B}(\vec{\rho})
$$

As noted in [3], this approximation may be reasonably good for many cases; it nevertheless constitutes a fundamental limitation in any analysis which docs not account for the directional dependence and potential rapid time dependencc of $T_{B}$.

With (4), (3) simplifies to

$$
T_{A}\left(\vec{\rho}_{A}\right)=\int_{E} d A \bar{G}\left(\vec{\rho}_{A}, \vec{\rho}\right) T_{B}(\vec{\rho})
$$

where position vector $\vec{\rho}_{A}$ is associated with direction $\hat{s}_{A}$, and $\bar{G}$ is given by

$$
\begin{aligned}
\bar{G}\left(\vec{\rho}_{A}, \vec{\rho}\right)= & \int_{-\infty}^{\infty} d t^{\prime} h\left(t-t^{\prime}\right) G\left(\hat{s}_{0}\left(t^{\prime}\right), \hat{s}\left(t^{\prime}\right)\right) \\
& \cdot\left[\frac{-\hat{s}\left(t^{\prime}\right) \cdot \hat{\rho}}{s^{2}\left(t^{\prime}\right)}\right]
\end{aligned}
$$

and describes the effective antenna gain function associated with the antenna temperature sample $T_{A}$. It should be noted that $\bar{G}$ takes into account the motion of the sensor platform, the characteristics of the antenna scan geometry, the effects of the radiometer low-pass filter, and, of course, the actual antenna gain function $G$.

To preserve the imaging properties of the antenna, the radiometer low-pass filter $\boldsymbol{h}$ must be selected to "match" the spatial frequency response of the antenna $G$ in (6). This may be accomplished by noting that the low-pass filter operates on the gain function $G$ primarily in the along scan direction. Thus, knowledge of the maximum spatial frequency sensed by $G$ in this direction and the antenna scan rate permits a determination of the maximum frequency of the waveform input to the filter. Of coursc, the filter should also have as narrow a passband as possible to minimize the effects of sensor noise. For an ideal lowpass filtcr with cutoff frequency equal to the product of the maximum spatial frequency sensed by the antenna $\left(\mathrm{km}^{-1}\right)$ and the scan ratc $(\mathrm{km} / \mathrm{s})$, the effective gain $\bar{G}$ (6) reduces to

$$
\bar{G}\left(\vec{\rho}_{A}, \vec{\rho}\right)=G\left(\hat{s}_{o}(t), \hat{s}\right)\left[\frac{-\hat{s} \cdot \hat{\rho}}{s^{2}}\right]
$$

which is simply the instantaneous antenna gain function at sample time $t$. A number of performance parameters must be addressed in the process of selecting a realizable low-pass filter that adequately approximates (7). These parameters include the distortion introduced by the filter due to nonuniform attenuation and group delay within the desired passband as well as the noise equivalent bandwidth of the filter. It should be noted that it is desirable 
to select $h$ so that (7) is maintained during the mcasurements. The resulting sampled data may be smoothed with a narrower filter during image processing if desired.

Now suppose that a set of antenna temperature samples $T_{A}\left(\vec{\rho}_{i}\right)$ at positions $\vec{\rho}_{i}, i=1, \cdots, N$ exist and that it is desired to estimate the antenna temperature at position $\vec{\rho}_{d}$ which lies within the region containing $\vec{\rho}_{i}$. The Backus-Gilbert theory may be applied to estimate the antenna temperature $\tilde{T}_{A}\left(\vec{\rho}_{d}\right)$ at $\vec{\rho}_{d}$ using a weighted linear combination of the neighboring antenna temperature samples,

$$
\tilde{T}_{A}\left(\vec{\rho}_{d}\right)=\sum_{i=1}^{N} a_{i} T_{A}\left(\vec{\rho}_{i}\right)
$$

where $\left\{a_{i}\right\}$ denotes the set of coefficients of the interpolation filter.

Substituting (5) into (8) and interchanging the sum and integral operations yields

$$
\tilde{T}_{A}\left(\vec{\rho}_{d}\right)=\int_{E} d A \bar{G}_{l}\left(\vec{\rho}_{d}, \ddot{\rho}\right) T_{B}(\vec{\rho})
$$

where $\bar{G}_{I}$ may be identified as the interpolated antenna gain function

$$
\bar{G}_{I}\left(\vec{\rho}_{d}, \vec{\rho}\right)=\sum_{i=1}^{N} a_{i} \bar{G}\left(\vec{\rho}_{i}, \vec{\rho}\right) .
$$

Now if $T_{A}\left(\vec{\rho}_{d}\right)$ represents the "true" antenna temperatures associated with $\vec{\rho}_{d}$, then the error in estimate (9) may be written as

$$
\begin{aligned}
e & =\left|T_{A}\left(\vec{\rho}_{d}\right)-\tilde{T}_{A}\left(\vec{\rho}_{d}\right)\right| \\
& =\left|\int_{E} d A\left[\bar{G}\left(\vec{\rho}_{d}, \vec{\rho}\right)-\bar{G}_{I}\left(\vec{\rho}_{d}, \vec{\rho}\right)\right] T_{B}(\vec{\rho})\right| .
\end{aligned}
$$

In the evcnt it is possible to select the set of $\left\{a_{l}\right\}$ so that $\bar{G}_{l}$ closely approximates $\bar{G}$, then $e$ will be minimized. If this is to occur, it is clear that $\bar{G}_{I}$ must represent a propcrly normalized antenna gain function so that

$$
\int_{E} d A \bar{G}_{I}=1
$$

As noted in [3], many criteria may be posed to minimize $e$, with perhaps the most useful ones presented in terms of the integral

$$
\begin{aligned}
Q_{d}= & \int_{F} d A\left[\bar{G}\left(\vec{\rho}_{d}, \dot{\rho}\right)\right. \\
& \left.-\sum_{i=1}^{N} a_{i} \bar{G}\left(\vec{\rho}_{i}, \vec{\rho}\right)\right]^{2} J\left(\vec{\rho}_{d}, \vec{\rho}\right)
\end{aligned}
$$

where $J$ is a specified function, and the set of weighting coefficients $\left\{a_{i}\right\}$ are selected to minimize $Q_{d}$ subject to the normalization constraint (12). $J$ may be selected to emphasize regions of importance within the integration. For example, in the event the interpolated gain function has undesirable sidelobe levels, then a functional form may be selected for $J$ which emphasizes the region of the sidelobes. Depending on the form selected, the reduction of the sidelobe level may result in some widening or slight distortion of the main beam. For the results presented herein, $J$ is set to unity.

The solution for the vector $\vec{a}$ with components $\left\{a_{i}\right\}$ may be written as

$$
\vec{a}=g-1\left[\vec{v}+\frac{\left(1-\vec{u}^{T} g^{-1} \vec{v}\right)}{\vec{u}^{T} g^{-1} \vec{u}} \vec{u}\right]
$$

where $g^{-1}$ is the inverse of matrix $g$ defined by components

$$
g_{i j}=\int_{E} d A \bar{G}\left(\vec{\rho}_{i}, \rho\right) \bar{G}\left(\vec{\rho}_{j}, \rho\right)
$$

and $\vec{u}$ and $\vec{v}$ are vectors with components

$$
\begin{aligned}
& \vec{u}_{i}=\int_{E} d A \bar{G}\left(\vec{\rho}_{i}, \dot{\rho}\right) \\
& \vec{v}_{i}=\int_{E} d A \bar{G}\left(\vec{\rho}_{i}, \vec{\rho}\right) \vec{G}\left(\ddot{\rho}_{d}, \vec{\rho}\right)
\end{aligned}
$$

and the superscript $T$ denotes transpose.

Note that in the event the interpolation point $\vec{\rho}_{d}$ coincides with one of the sampled points $\vec{\rho}_{k}$, the interpolated temperature reduces to the sampled temperature at $\vec{\rho}_{k}$ since, in this case, $Q_{d}$ vanishes with $a_{k}=1$ and $a_{i}=0$ $(i \neq k)$.

The rcadcr may recognize that criterion (13) with constraint (12) is equivalent to an unbiased minimum mean squared estimator of $T_{A}$ for the situation in which $T_{B}$ is treated as a homogeneous random process [8] having a uniform power spectral density. That is, expressing $T_{B}$,

$$
T_{B}=\left\langle T_{B}\right\rangle+\delta T_{B}
$$

where $\left\langle>\right.$ denotes the expectation operator and $\delta T_{B}$ is the zero mean random component of $T_{B}$, then under the above assumption for $T_{B}$, the mean squared error of $e$ (11) may be written as

$$
\begin{aligned}
e^{2}= & \left\langle T_{B}\right\rangle^{2}\left\{\int_{E} d A\left[\bar{G}-\bar{G}_{I}\right]\right\}^{2} \\
& +\left\langle\delta T_{B}^{2}\right\rangle \int_{t} d A\left[\bar{G}-\bar{G}_{I}\right]^{2} .
\end{aligned}
$$

The first term on the right-hand side of (19) is the basis of the estimator and it vanishes if (12) holds. The minimization of the second term is equivalent to minimizing (13) with $J=1$.

Finally. it should be noted that the instrument noise appearing in the sampled antenna temperatures propagates to the interpolated temperature (9). As presented in [3], a minimization criteria may be established to include a lin- 
car weighted sum of $Q_{d}$ and the variance of the instrument noise in the estimate (9) (see [13, eq. (13)]). The approach taken here is to select $a_{i}$ which minimize $Q_{d}$ subject to (12) without consideration of instrument noise. However, it is important to examine the resulting variance of the interpolated antenna temperatures $e^{2}$ for each set of coefficients:

$$
e^{2}=\vec{a}^{\top} E \vec{a}
$$

where $E$ is the covariance matrix of the instrument noise process appearing in the sampled antenna temperatures, and is a function of system noise temperature, predetection bandwidth, and receiver low-pass filter. When the noise in each sample is uncorrelated and equal, then $e^{2}$ is proportional to the sum of the squares of $\boldsymbol{a}_{i}$ times the variance of the instrument noisc.

\section{Exampi,es}

As an application of the above theory, consider the problem of interpolating the satellite microwave radiometer data collected by the Special Sensor Microwave/Imager $(\mathrm{SSM} / \mathrm{I})$. The SSM/I is a seven-channel four-frequency linearly polarized conically scanning microwave radiometer on board the joint United States Air Force/ Navy Defense Meteorological Satellite Program (DMSP) spacecraft. The satellite operates in a circular sun-synchronous near polar orbit at a nominal altitude of $833 \mathrm{~km}$ and an inclination of $98.8^{\circ}$ and orbit period of $102 \mathrm{~min}$. A description of the SSM/I instrument and the environmental products retrievablc from its data is given in the SSM/I User's Guide [9] and the SSM/I Cal/Val Final Report [10]. Suffice it to say that the antenna system, which consists of a single offsct parabolic reflector illuminated by a single wide-band multichannel feedhom, produces seven nearly circular coaxial beams with $3 \mathrm{~dB}$ beamwidths of $1.9^{\circ}, 1.06^{\circ}$, and $0.4^{\circ}$ with dual linear polarizations at channel frequencies of $19.35,37.0$, and 85 $\mathrm{GHz}$, respectively, and $1.6^{\circ}$ with single polarization at $22.235 \mathrm{GHz}$. During the conical scan, the antenna beams trace out a circular arc on the earth's surface at a constant nominal $53.1^{\circ}$ incidence angle across a swath width of $1400 \mathrm{~km}$. The antenna scan rate of $30 \mathrm{rpm}$ and channel data sampling rates result in a $12.5 \mathrm{~km}$ sampling grid for the highest resolution $85 \mathrm{GHz}$ channels along the circular arc, and a $12.5 \mathrm{~km}$ sampling increment between successive scans along the direction of the subsatellite ground track. For the lower resolution channels, the spatial sampling grid is $25 \mathrm{~km}$ along the circular arc and $25 \mathrm{~km}$ between successive scans.

The conical scan geometry is needed to retrieve environmental products. At the same time this geometry provides a uniform antenna gain function for each sample antenna temperature. However, the conical scan introduces a dcgree of complexity in the relative geometry of the sampled temperatures when viewed in a coordinate system on the earth's surface. For example, Fig. 2 illustrates the relative positions of the $85 \mathrm{GHz}$ samples for regions near the start of scan, middle of scan, and end of scan in a latitude/longitude coordinate system. The circles identify the $3 \mathrm{~dB}$ contour of the antenna gain function projected on the earth's surface and shows the variation of the relative sample geometry and beam overlapping across the swath.

It should be noted that the SSM/I employs an integrate and dump filter for the radiometer low-pass filter which, in accordance with (6), increases the cffective antenna beam diameter primarily in the along scan direction. An integration time of $4.22 \mathrm{~ms}$ is used at $85 \mathrm{GHz}$ and translates to an azimuthal integration region of $0.8^{\circ}$. For the remaining channels, thc integration times are $8.44 \mathrm{~ms}$ with an $1.6^{\circ}$ azimuthal region. The $85 \mathrm{GHz}$ data are sampled at $4.22-\mathrm{ms}$ interval and the remaining channels at 8.44 ms. The increase in beam diamctcr at $85 \mathrm{GHz}$ is just enough to causc the projected $3 \mathrm{~dB}$ contour to be nearly circular. The $3 \mathrm{~dB}$ contours for the remaining channels are considerably more elliptical with corresponding more beam overlapping of $3 \mathrm{~dB}$ contours than shown in Fig. 2 .

Since thc integrate and dump filter is not matched to the antenna gain function, the full resolution of the gain function (7) is not realized in the along scan direction. The loss in resolution is significant at both 37 and $85 \mathrm{GHz}$ and was implemented in the SSM/I to meet the spacecraft data rate of $3.6 \mathrm{~kb} / \mathrm{s}$. The results presented below apply to the effective gain factor (6) with the integrated and dump filter.

To illustrate the efficacy of the interpolation process presented above, consider the problem of interpolating the $85 \mathrm{GHz}$ antenna temperature at a point located midway between successive scans and midway between along scan samples. The point marked by " $\mathrm{X}$ " in Fig. 2 identifies the interpolation point of interest for each of the three scan regions. Since the antenna gains are reasonably directive, the set of neighboring sampled antenna tcmperatures used in the interpolation may be restricted to 3-4 samples in each direction about point $X$. For definiteness, consider a four $x$ four array. From Fig. 2 it is clear that the neighboring samples (lying within the dashed line) form a square array near the subsatellite track and parallelogram arrays near the edges of the swath.

Although the "true" antenna temperature at point $\mathrm{X}$ is not available, the appropriateness of the interpolated temperature may be judged on the basis of how closely the interpolated antenna gain function $\bar{G}_{l}$ (9) approximates the "desired" antenna gain function $\bar{G}$. In the results presented below, the computation of $\bar{G}(6)$, and, hence $\bar{G}_{/}$, include simulations of the satellite motion over a spherical earth, the SSM/I scan geometry, the radiometry integrate and dump low-pass filter, and actual antenna gain functions as determined from measurements on an antcnna range.

Figs. 3-5 present the $85 \mathrm{GHz}$ (horizontal polarization) interpolated antenna gain $\bar{G}_{I}$ (solid line) and the desired 


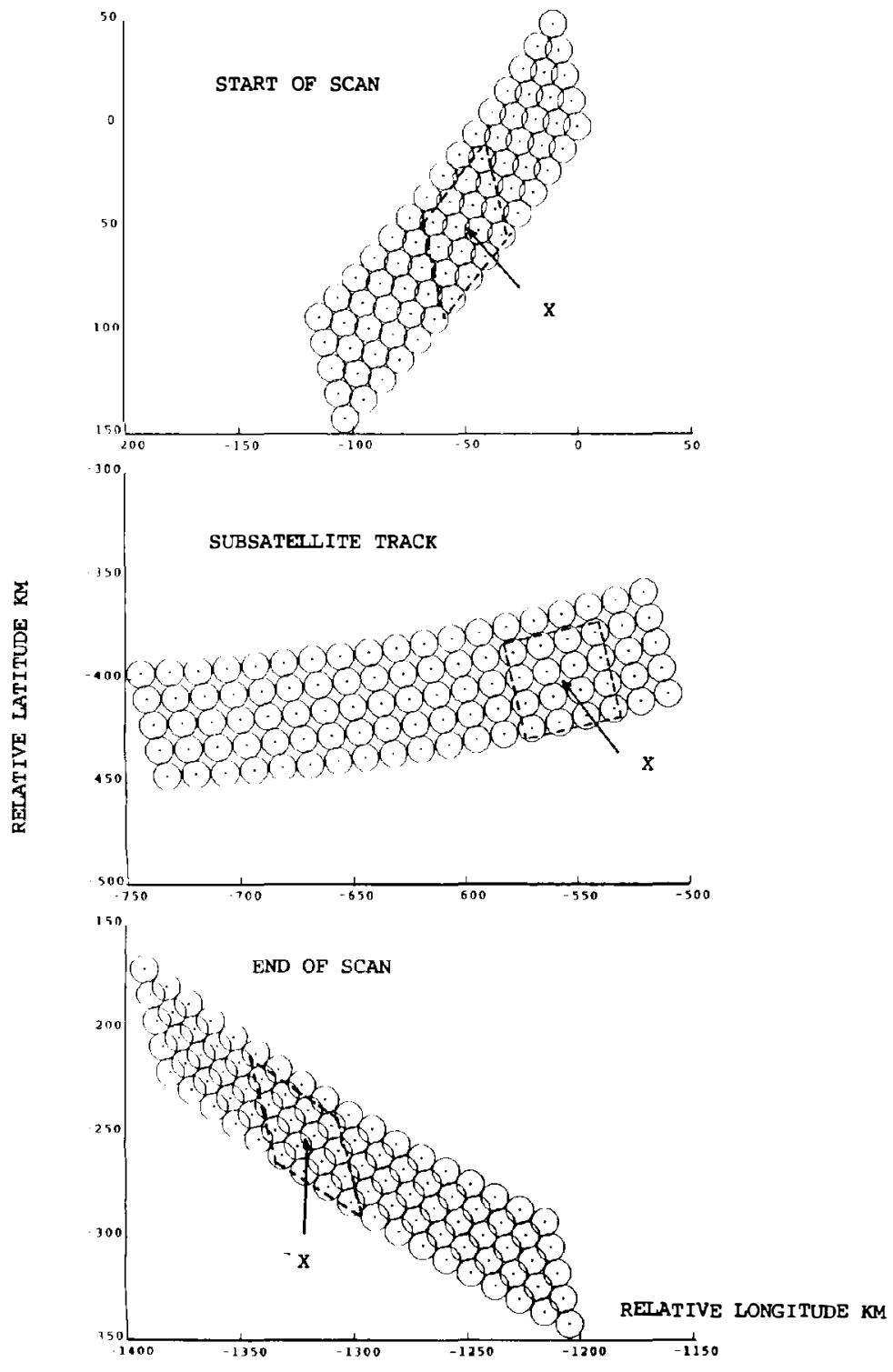

Fig. 2. SSM/I $85 \mathrm{GHz}$ sample geometry with interpolation point marked with " $\mathrm{X}$. "

or "true" antenna gain $\bar{G}$ (dashed line) along the directions defined by the principal $E$ - and $H$-planes passing through the interpolation point for the three scan regions noted. The $E$-plane lies in the along view direction and the $H$-plane lies in the along scan direction. Note that better agreement occurs between the interpolated and desired antenna patterns near the edges of the scan region than near the subsatellite track. Figs. 6 and 7 present contours of the interpolated antenna gain functions and again illustrate that better agreement occurs near the edges of the scan region. Table I presents the interpolation coefficients a for the three cases associated with Figs. 3-5. The coefficients may be identified with the $4 \times 4$ pixels shown in Fig. 2 (bottom to top, right to left). As expected, the coefficients are largest for the pixels nearest the interpolation point with. in many cases, important contributions arising from the remaining pixels.

The reason for the improved behavior near the scan edges may be seen by noting that the $12.5 \mathrm{~km}$ sampling grid used for the $85 \mathrm{GHz}$ data is not consistent with the spatial frequency response of the antenna. To verify this, suppose that the number of samples were increased by a 

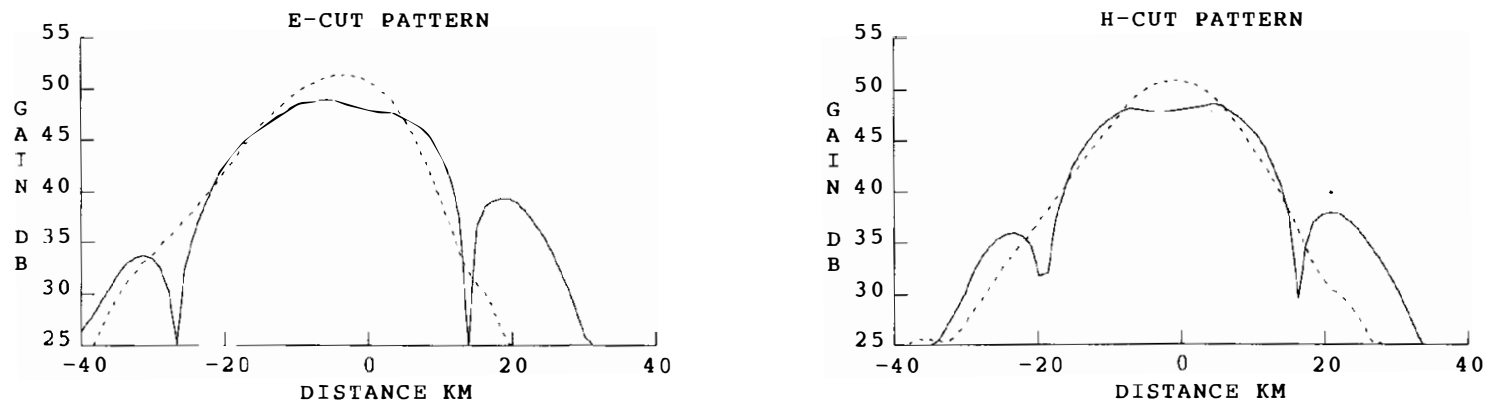

Fig. 3. $85 \mathrm{GHz}$ true (dashed) and interpolated (solid) patterns at subsatellite track.
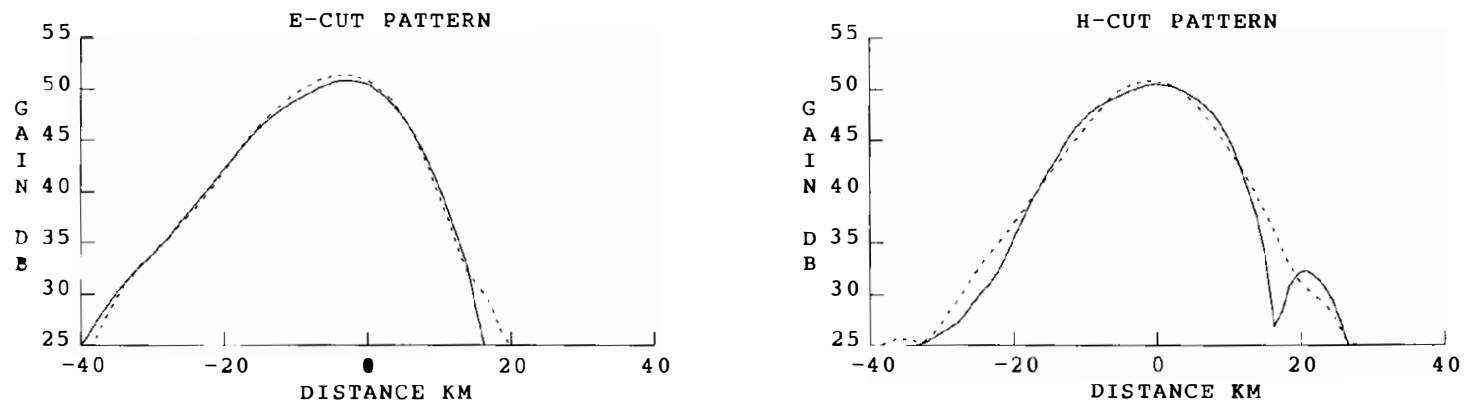

Fig. 4. $85 \mathrm{GHz}$ true (dashed) and interpolated (solid) patterns near start of scan.
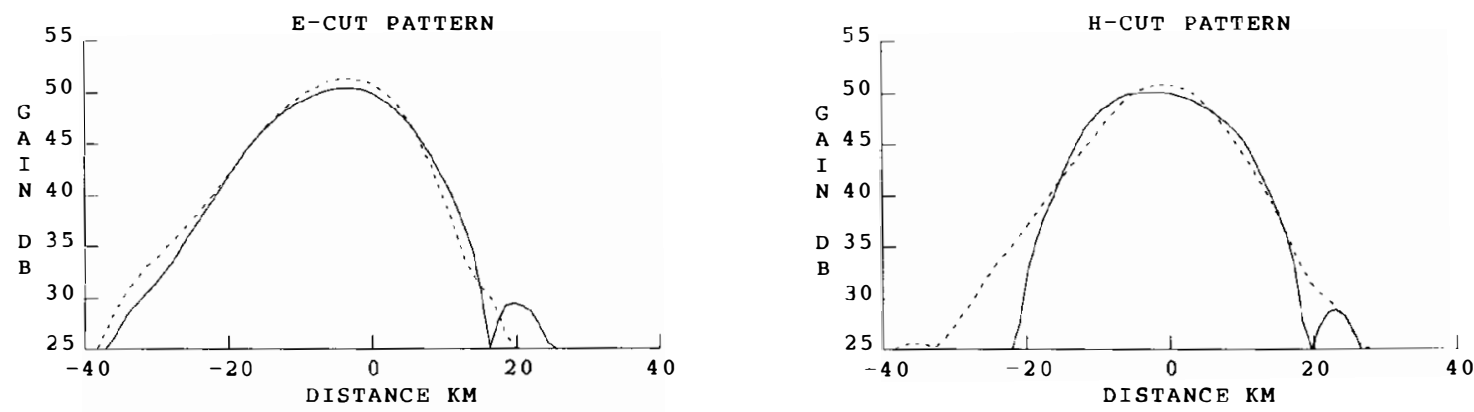

Fig. 5. $85 \mathrm{GHz}$ true (dashed) and interpolated (solid) patterns near end of scan.
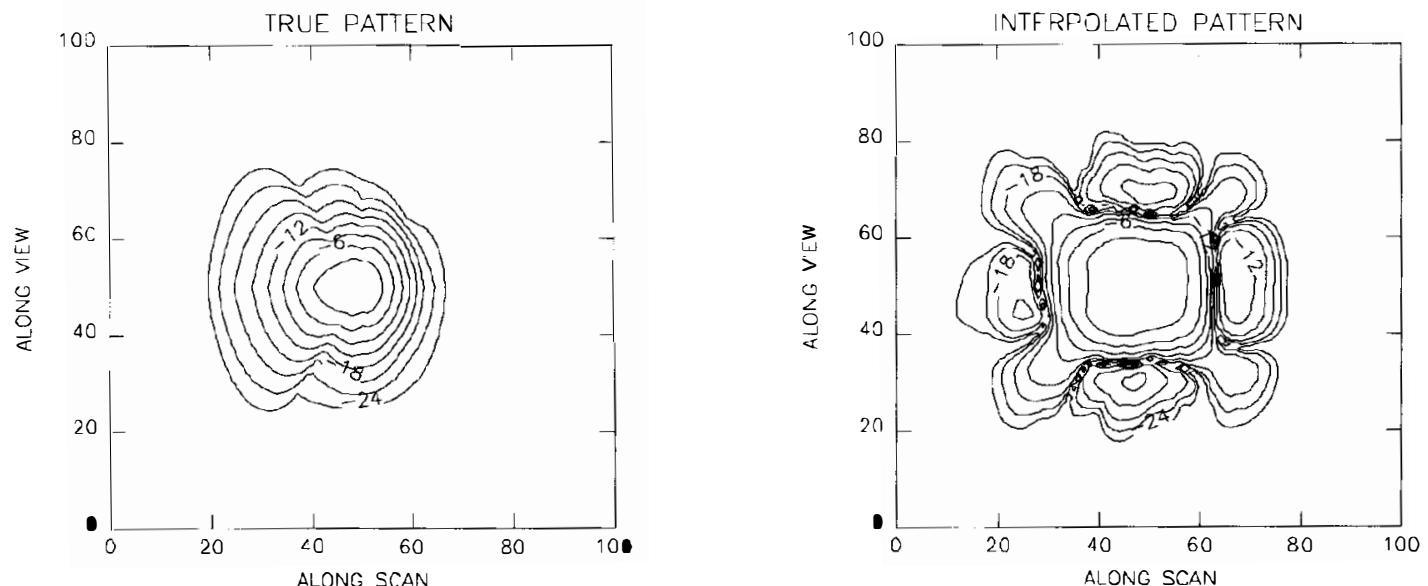

Fig. $6.85 \mathrm{GHz}$ contours at subsatellite track. 

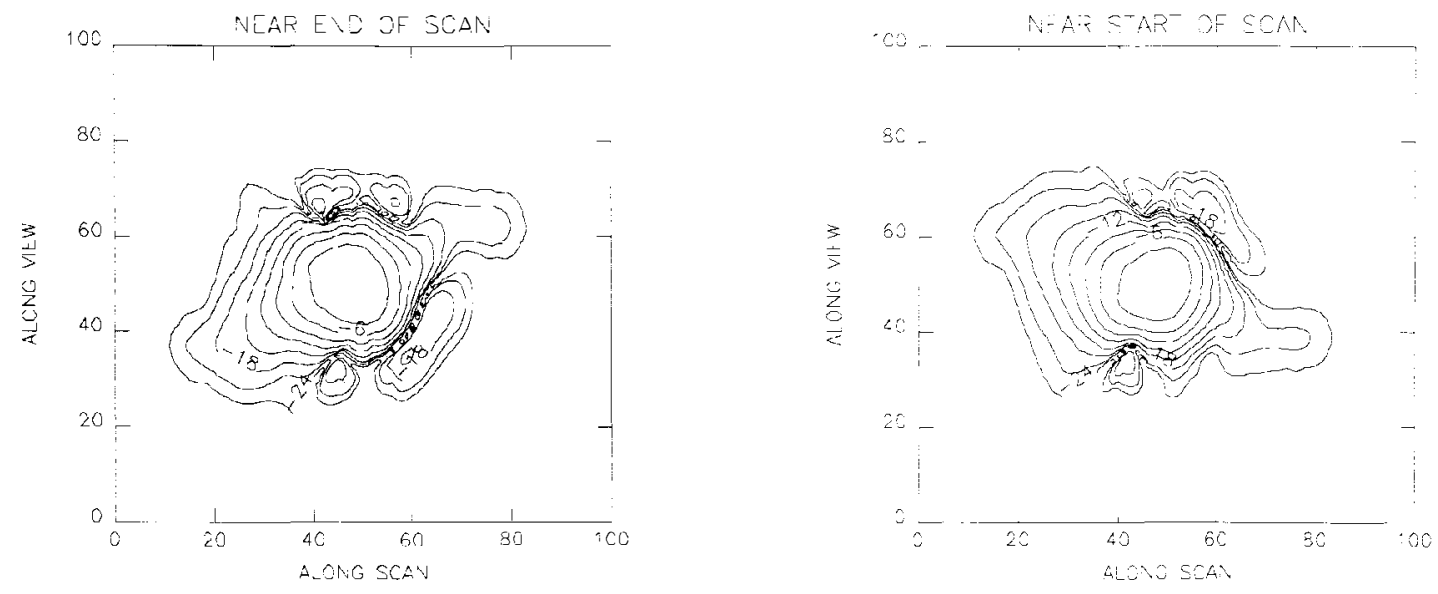

Fig. 7. $85 \mathrm{GHz}$ interpolated contours.

TABLE I

Interpelation Colfficients Associated with the Figures Noted. Coefficients Apply for Pixel Geometry of Fig. 2 (Bettom to Tup, RIGHT TO LEFT)

\begin{tabular}{|c|c|c|c|}
\hline \multicolumn{4}{|c|}{$\begin{array}{l}\text { Figure 3: } 85 \text { GHz Near Subsatellite Track } \\
\text { (RMS noise factor }=0.71 \text { ) }\end{array}$} \\
\hline $\begin{array}{r}0.0167 \\
-0.0489 \\
-0.0493 \\
0.0156\end{array}$ & $\begin{array}{r}-0.0647 \\
0.3445 \\
0.3483 \\
-0.0622\end{array}$ & $\begin{array}{r}-0.0625 \\
0.3528 \\
0.3400 \\
-0.0643\end{array}$ & $\begin{array}{r}0.0142 \\
-0.0516 \\
-0.0465 \\
0.0179\end{array}$ \\
\hline \multicolumn{4}{|c|}{$\begin{array}{l}\text { Figure 4: } 85 \mathrm{GHz} \text { Near start of Scan } \\
\text { (RMS noise factor }=0.87 \text { ) }\end{array}$} \\
\hline $\begin{array}{r}-0.1170 \\
-0.0035 \\
0.0021 \\
0.0039\end{array}$ & $\begin{array}{r}-0.0034 \\
0.6151 \\
0.0092 \\
0.0015\end{array}$ & $\begin{array}{r}0.0015 \\
0.0098 \\
0.5884 \\
-0.0033\end{array}$ & $\begin{array}{r}0.0039 \\
0.0022 \\
-0.0040 \\
-0.1064\end{array}$ \\
\hline \multicolumn{4}{|c|}{$\begin{array}{l}\text { Figure 5: } 85 \text { GHz Near End of Scan } \\
\text { (RMS noise factor }=0.87 \text { ) }\end{array}$} \\
\hline $\begin{array}{r}0.0038 \\
0.0021 \\
-0.0037 \\
-0.1065\end{array}$ & $\begin{array}{r}0.0015 \\
0.0091 \\
0.5887 \\
-0.0030\end{array}$ & $\begin{array}{r}-0.0029 \\
0.6149 \\
0.0085 \\
0.0016\end{array}$ & $\begin{array}{r}-0.1169 \\
-0.0033 \\
0.0020 \\
0.0038\end{array}$ \\
\hline \multicolumn{4}{|c|}{$\begin{array}{l}\text { Figure 8: } 85 \mathrm{GHz} \text { Near Subsatellite Track } \\
\text { with Nyquist Sampling (RMS noise }=0.74 \text { ) }\end{array}$} \\
\hline $\begin{array}{r}0.0171 \\
-0.0622 \\
-0.0712 \\
0.0202\end{array}$ & $\begin{array}{r}-0.0539 \\
0.3448 \\
0.3681 \\
-0.0626\end{array}$ & $\begin{array}{r}-0.0625 \\
0.3694 \\
0.3444 \\
-0.0559\end{array}$ & $\begin{array}{r}0.0187 \\
-0.0705 \\
-0.0632 \\
0.0192\end{array}$ \\
\hline \multicolumn{4}{|c|}{$\begin{array}{l}\text { Figure 10: } 19 \text { GHz Near Subsatellite Track } \\
\text { (RMS noise factor }=0.74 \text { ) }\end{array}$} \\
\hline $\begin{array}{r}0.0222 \\
-0.0896 \\
-0.0608 \\
0.0217\end{array}$ & $\begin{array}{r}-0.0576 \\
0.3786 \\
0.3366 \\
-0.0508\end{array}$ & $\begin{array}{r}-0.0508 \\
0.3545 \\
0.3607 \\
-0.0585\end{array}$ & $\begin{array}{r}0.0189 \\
-0.0765 \\
-0.0746 \\
0.0261\end{array}$ \\
\hline \multicolumn{4}{|c|}{$\begin{array}{l}\text { Figure 12: } 37 \mathrm{GHz} \text { Smoothed to } 19 \mathrm{GHz} \text { Near } \\
\text { Subsatellite Track (RMS noise }=0.42 \text { ) }\end{array}$} \\
\hline $\begin{array}{l}-0.0031 \\
-0.0092 \\
-0.0105 \\
-0.0003\end{array}$ & $\begin{array}{l}0.0609 \\
0.1921 \\
0.2161 \\
0.0663\end{array}$ & $\begin{array}{l}0.0501 \\
0.1932 \\
0.2087 \\
0.0631\end{array}$ & $\begin{array}{l}-0.0011 \\
-0.0127 \\
-0.0112 \\
-0.0024\end{array}$ \\
\hline
\end{tabular}

factor of two in both the along scan and along track directions, i.e., a sampling of $6.25 \mathrm{~km}$ along the circular arc and $6.25 \mathrm{~km}$ between successive scans at the subsatellite track. Then recomputing the interpolation antenna gain functions at a point midway between the new along scan samples and midway between new successive scans reveals a significant improvement over the rcsults of Fig. 3. This may be seen from results presented in Fig. 8 which show the interpolated and desircd antenna patterns in the principal $E$ - and $H$-planes. The degree of improvement may also be seen in Fig. 9 which shows the contours of the interpolated gain function. Thus, the better agreement between the interpolated and derived antenna gain functions near the scan edges in Figs. 4 and 5 is due to the closer spacing of the sampled antenna temperatures. Comparison of the results shown in Figs. 3 and 6 to those of Figs. 8 and 9 clearly demonstrates the importance of proper data sampling. Without Nyquist sampling, attempts to intcrpolate, resample, or, for that matter, perform most image analysis functions will result in a loss of spatial resolution. In addition, depending on the spatial frequency spectra of the earth's brightness temperature field being imaged, processing may introduce artifacts in the images commonly referred to as aliasing errors [11]. Table I presents the set of coefficients for the interpolation filter of Figs. 8 and 9 with the corresponding noise amplification factor.

A situation similar to that presented above for the 85 $\mathrm{GHz}$ data exists when intcrpolating the $37 \mathrm{GHz}$ antenna temperatures. The problem stems again from a spatial sampling grid of $25 \mathrm{~km}$ that is not consistent with the spatial frequency response of the antenna at $37 \mathrm{GHz}$. The grid is approximately twice as large as needed to satisfy the Nyquist criterion. However, the interpolation of the $19 \mathrm{GHz}$ data reveal that the interpolated gain functions exhibit reasonably good agreement with the desired gain. For example, Fig. 10 presents results obtained using a 4 $\times 4$ array of samples to interpolate the $19 \mathrm{GHz}$ (vertical polarization) at a point having the same relative position midway between along scan samples and midway be- 

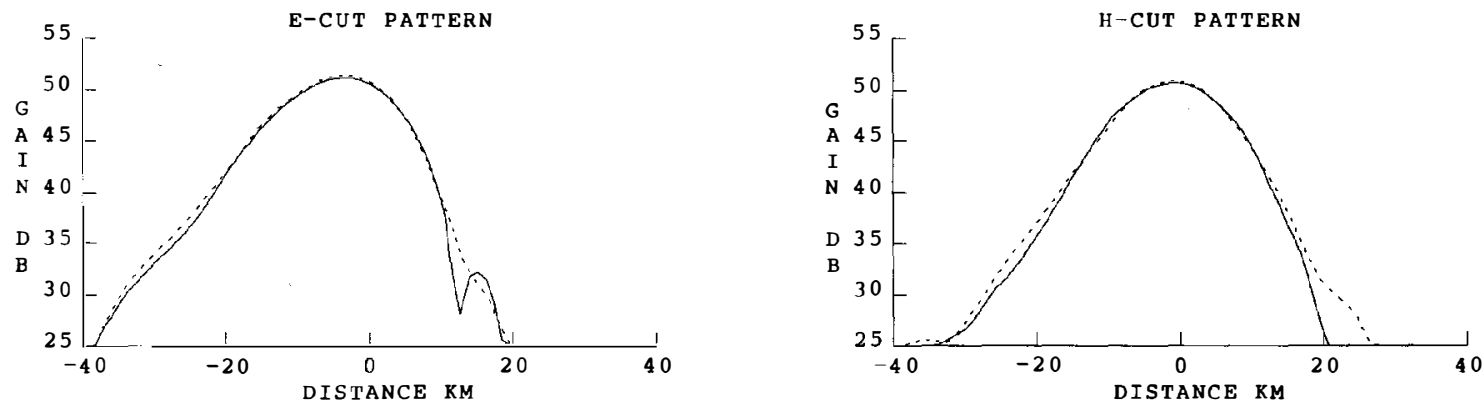

Fig. 8. $85 \mathrm{GHz}$ true (dashed) and interpolated (solid) patterns at subsatellite track with Nyquist sampling.

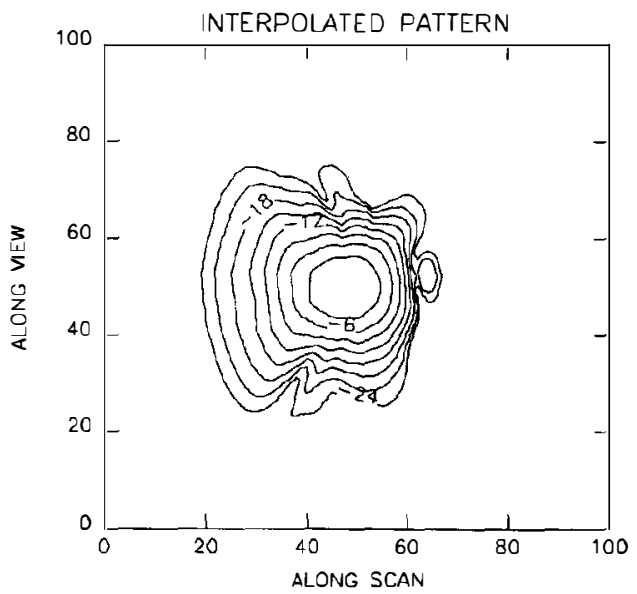

Fig. 9. $85 \mathrm{GHz}$ contours at subsatellite track with Nyquist sampling.
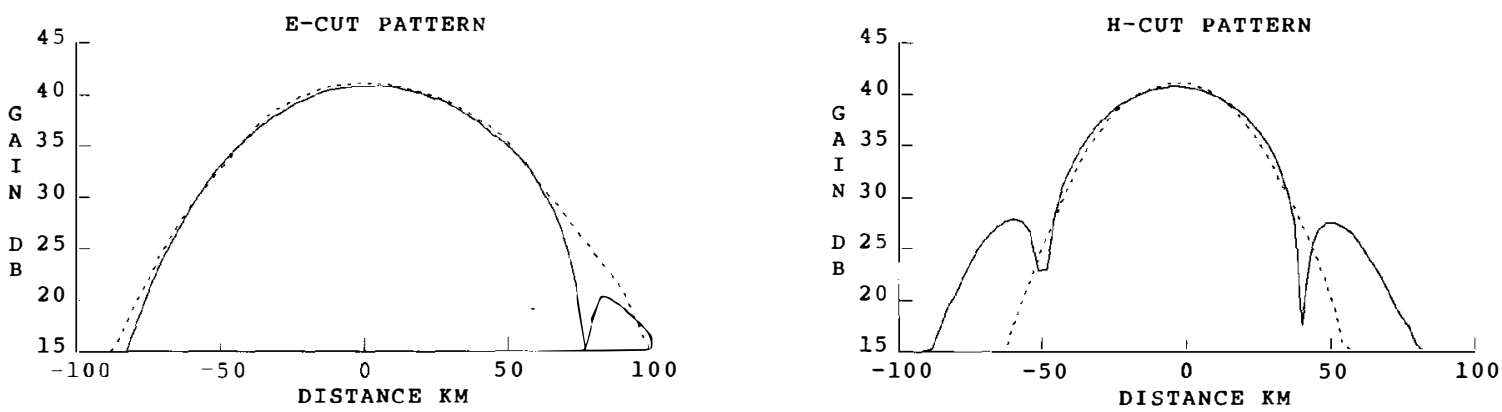

Fig. 10. $19 \mathrm{GHz}$ true (dashed) and interpolated (solid) patterns at subsatellite track.

tween successive scans as shown in Fig. 2 near the subsatellite track. Fig. 11 presents the contours of the interpolated and desired antenna gain functions. Note the agreement is quite good over most of the patterns in Fig. 10 except for the sidelobes appearing in the $\boldsymbol{H}$-cut pattern. This arises from the fact that the antenna gain function is considerably narrower in the along scan direction, and consequently the $25 \mathrm{~km}$ sampling increment is too large to meet the Nyquist criteria in this direction. As noted in the discussion following (13), the level of the sidelobes may be reduced, if desired, by selecting a form for $J$ which emphasizes the region containing the sidelobes. Table I presents the interpolation coefficients for case of Figs. 10 and 11 .

It is interesting to note that the interpolation process may be combined with the task of reducing the resolution of the 37 or $85 \mathrm{GHz}$ data to match the resolution of the $19 \mathrm{GHz}$ data. That is, if the function $\bar{G}\left(\vec{\rho}_{d}, \vec{\rho}\right)$ appearing in (13) is associated with the $19 \mathrm{GHz}$ antenna gain function, and the functions $\bar{G}\left(\vec{\rho}_{i}, \vec{\rho}\right)$ for $i=1, \cdots, N$ are 

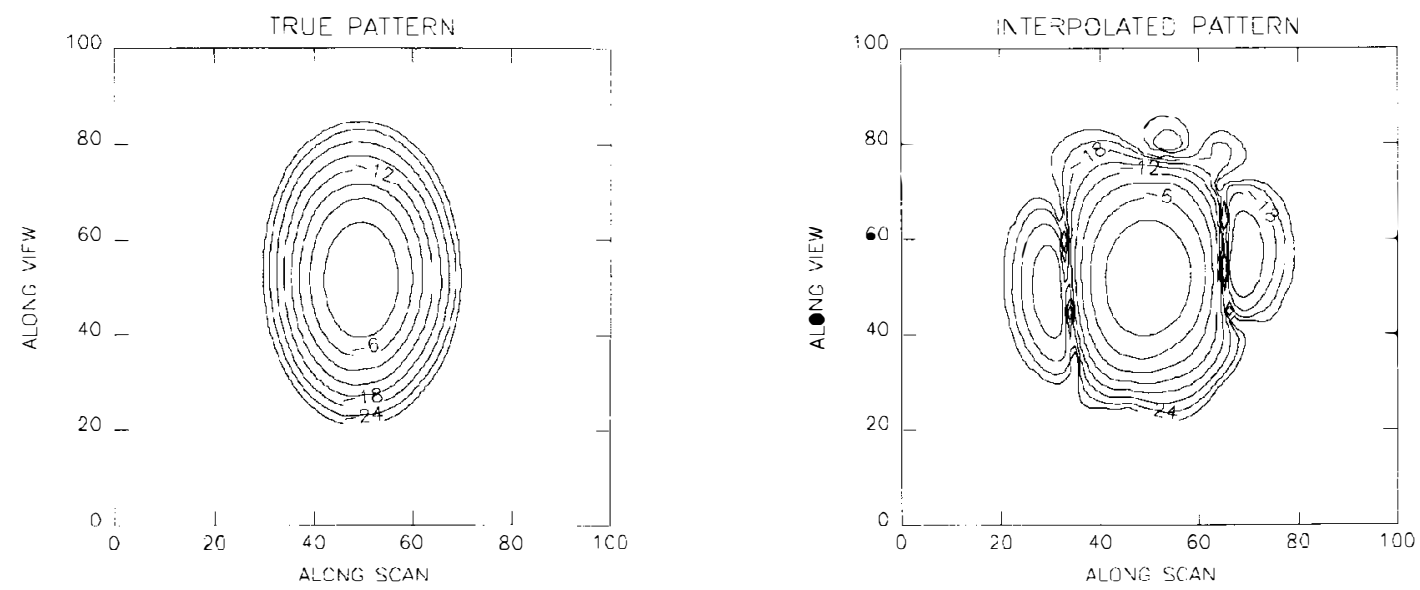

Fig. 11. $19 \mathrm{GHz}$ contours at subsatellite track.
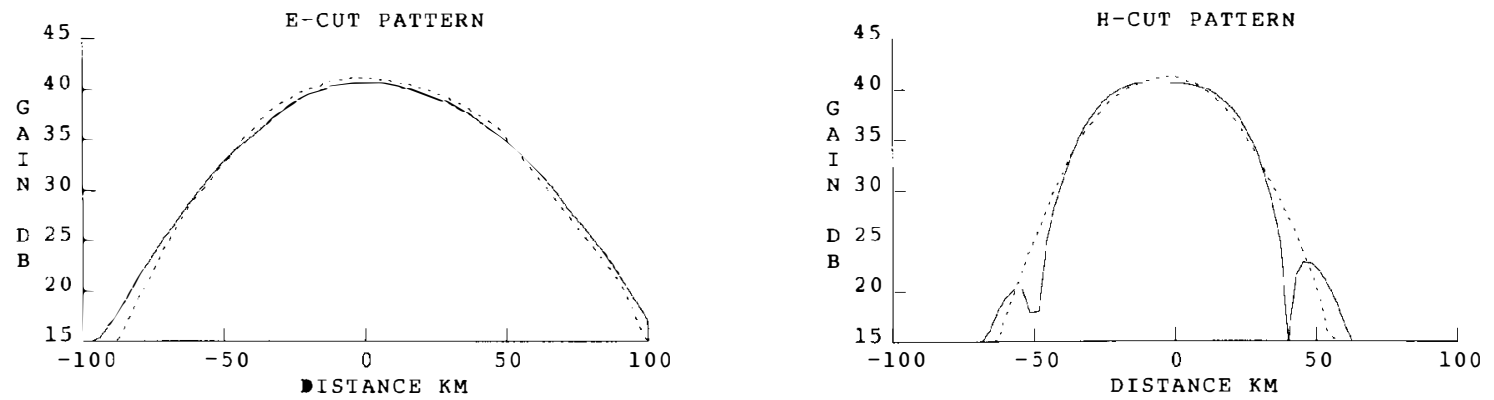

Fig. 12. $19 \mathrm{GHz}$ true (dashed) and $37 \mathrm{GHz}$ interpolated (solid) patterns at subsatellite track.

associated with the 37 or $85 \mathrm{GHz}$ antenna gain functions, then the solution for the coefficients $\vec{a}$ (14) will provide an interpolated 37 or $85 \mathrm{GHz}$ antenna gain which closely approximates the derived $19 \mathrm{GHz}$ antenna gain function. For example, Fig. 12 presents the interpolated $37 \mathrm{GHz}$ antenna patterns (horizontal polarization) using a $4 \times 4$ array to interpolate and match the $19 \mathrm{GHz}$ sample (vertical polarization) at a point $X$ located midway between the along scan samples and midway between successive scans near the subsatellite track (this is the same point used in Figs. 10 and 11). Fig. 13 shows the contours of the interpolated antenna gain and compares reasonably well with the $19 \mathrm{GHz}$ surface shown in Fig. 11. Table I presents the coefficients of the interpolation filter with the associated noise factor.

As a final illustration of the benefits of the interpolation process, consider the Mercator projection of an $85 \mathrm{GHz}$ horizontally polarized radiometric image of the East Coast of the United States presented in Fig. 14. The image was created with the interpolation approach presented herein using a $4 \times 4$ array of neighboring sampled antenna temperatures at each point of interpolation. As a consequence, the weighting coefficients varied across the swath as well as between successive scans, but because of the

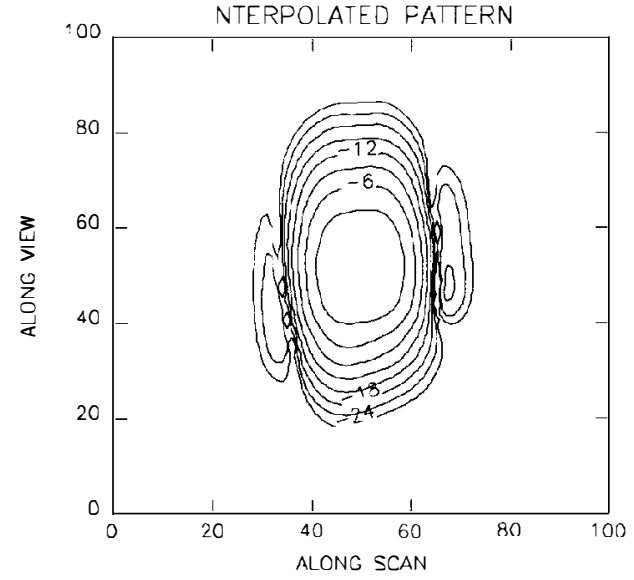

Fig. 13. $37 \mathrm{GHz}$ contours at subsatellite track matched to $19 \mathrm{GHz}$ patterm

periodic nature of the scan motion, the weighting coefficients repeat between successive scans. The total number of samples was increased by a factor of four in both the along track and along scan directions. The data were taken by the SSM/I on a descending pass for orbit number 4623 


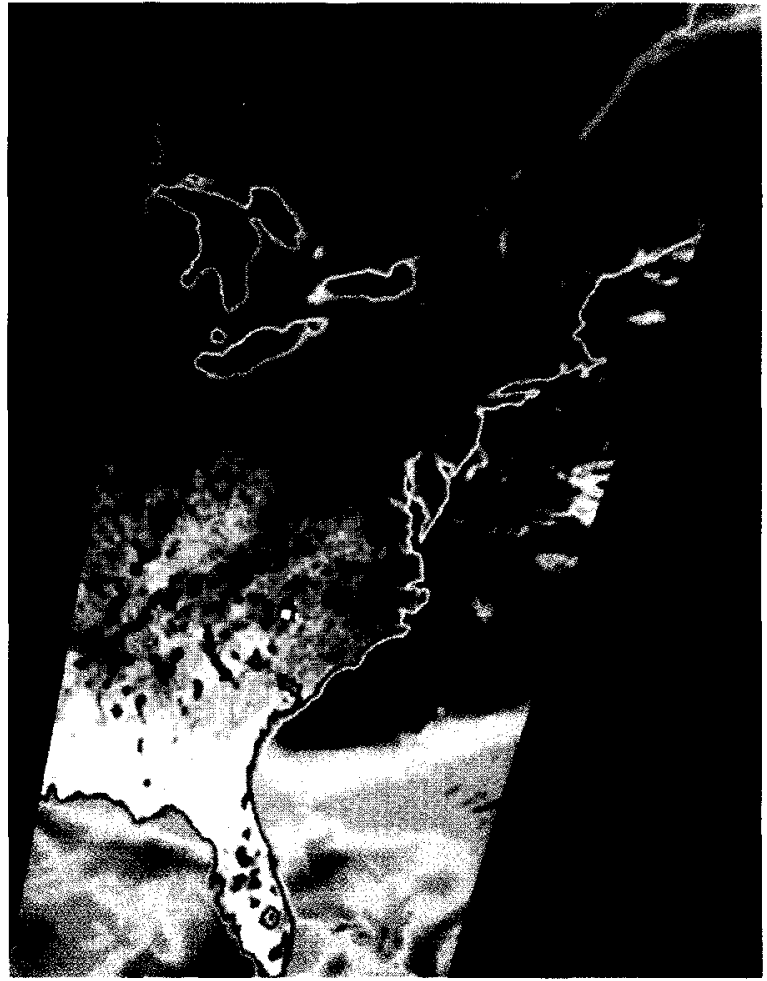

Fig. 14. $85 \mathrm{GHz}$ image (horizontal polarization) with interpolation; orbit 4263. April 17. 1988

in mid-April 1988. A continuous color bar (blue-grecnred-whitc) spans 250 levels and covers antenna temperatures from $150 \mathrm{~K}$ to $280 \mathrm{~K}$.

For comparison, Fig. 15 presents an image of the same scene of Fig. 14 generated with a commonly used simple pixcl replication filter. Although this procedure permits a more rapid means of image construction than obtainable with the "optimum" interpolation filter described herein, Fig. 14 clcarly shows a considerable improvement over Fig. 15 in image quality. This is especially noticeable in the identification of fine details along the Atlantic coastline, the shorcline regions around the Great Lakes. and along the cloud boundaries in the lower part of the image.

Fig. 14 presents an image that attempts to prescrve the spatial resolution of the original sampled radiometric data. For some situations, this may be a highly desirable feature. For example. imagery similar to that of Fig. 14 has been employed with an overlay of a world coastline database to analyze and correct geolocation errors appearing in the SSM/I data [12]. In addition, the interpolation process described herein has been used to create imagcry of tropical cycloncs, ocean surface wind fields [13], and sea ice boundaries [10] for detailed analyses, as well as for comparisons of polar sea ice concentration maps with visual and infrared imagery from the Advanced Very High Resolution Radiometer (AVHRR) [14].

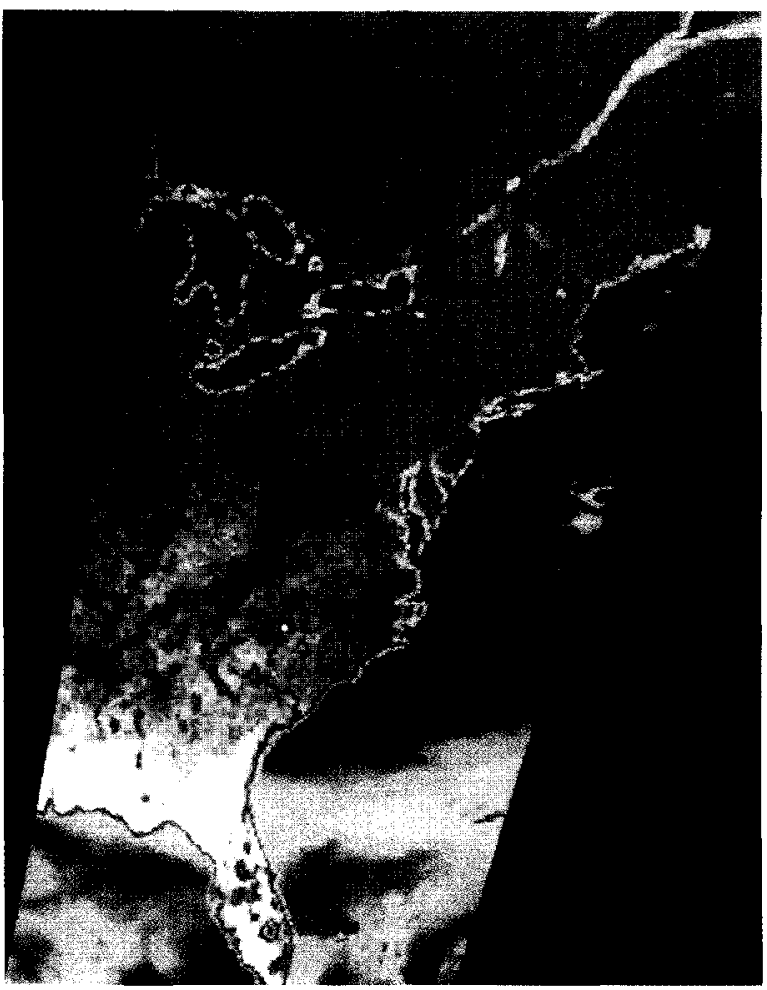

l.ig. 15. $85 \mathrm{GHz}$ image (horizontal pularization) with simple pixel replication filter; orbit 4263. April 17, 1988

\section{Conclusions}

An optimum interpolation technique has been presented to interpolate, resample, and smooth imaging microwave radiometer data. The technique was applicd to the satellite Spccial Sensor Microwave/Imager data, and results were presented which show the efficacy of the methodology in attempting (o) preserve the spatial resolution of the originally sampled data and, hence. in enhancing the quality of the imagery. It is expected that proper filtering, sampling, and interpolation of satellite data will continue to receive incrcasing attention as analysts extract information from high-resolution microwave radiometric imagery.

\section{ACKNOWLEDGMENT}

The author wishes to express appreciation to R. Conway, D. Conway, E. Overton, and D. Spangler for assistance with the computer programming and image processing; to Dr. J. Hollinger for valuable discussions, encouragcment, and support; and to M. Spangler for her patience in preparing this manuscript. The author also thanks the reviewers of this paper for valuable suggestions.

\section{REFERENCES}

[1] H. C. Ko. "Radio-telescope antenna parameters." IEEE Trans. An tennas Propagat.. vol. AP-12. pp. 891-398. Dec. 1964 
[2] H. J. Landau, "Sampling, data transmission, and Nyquist ratc," Proc. IEEE, vol. 55, pp. 1701-1706, Oct. 1967.

[3] A. Stogryn, "Estimates of brightness temperatures from scanning radiometer data." IEEE Trans. Antennas Propagat., vol. AP-26, pp.720-726, Sept. 1978.

[4] A. S. Milman, "Antenna pattern correction for the Nimbus-7 SMMR," IEEE Trans. Geosci. Remote Sensing, vol. GE-24, pp. 212219, Mar. 1986.

[5] G. J. Buck and J. J. Gustincic, "Resolution limitations of a finite aperture," IEEE Trans. Antennas Propagat., vol. AP-15, pp. 376$381,1967$.

[6] R. B. Frieden, "Band-unlimited reconstruction of optical objects and spectra," J. Opt. Soc. Amer., vol. 57. no. 8, pp. 1013-1019. 1967.

[7] T. S. Huang et al., "Image processing," Proc. IEEE, vol. 59. pp. 1586-1609, 1971

[8] V. I. Tatarski, Wave Propagation in a Turbulent Medium. New York: McGraw-Hill, 1961, p. 15.

[9] J. Hollinger et al., Special Sensor Microwavellmager User's Guide. Naval Res. Lab., Washington, DC, 1987.

[10] J. Hollinger et al., "DMSP Special Sensor Microwave/Imager Calibration/Validation," Final Rep., Vol. I, Naval Res. Lab., Washington, DC, 1989.

[11] K. R. Castleman, Digital Image Processing. Englcwood Cliffs, NJ Prentice-Hall, 1979, pp. 230-234.

[12] G. A. Poe and R. W. Conway, "A study of the geolocation crrors of the SpccialSensor Microwave/Imager (SSM/I), " IEEE Trans. Geosci. Remote Sensing, this issue. pp. 791-799.

[13] G. Sandlin and D. Spangler, "'SSM/I imagery, center-fixes and wind fields for tropical cyclones." Naval Res. Lab. Rcmote Sensing Branch Rep., Washington, DC. 1989

[14] J. Hawkins and A. Chase, "Operational polar remotc sensing data fusion," Trans. Amer. Geophys. Union. submitted for publication.

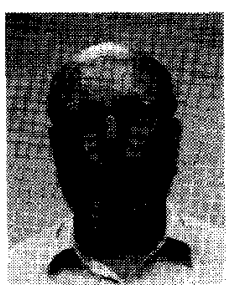

Gene A. Poe was born in Tulsa, OK, on March 29. 1942. He received the B.A. and M.S. degrees in electrical engineering from the University of Califomia. Berkeley, in 1964 and 1965

From 1986 through 1989 he worked in the Space Sensing Branch of the Naval Research Laboratory on microwave radiometric modeling and analysis of SSM/I data, as well as on radiometric target detection problems. From 1978 through 1983 he was employed by the Space and Communication Group of Hughes Aircraft Company. where he worked on microwave sensor design and retrieval algorithms of remotely sensed data. He is currently employed by Aerojet ElectroSystems Company, Azusa, CA, where he is working on advanced microwave radiometric sensor systems. 